\title{
Renormalized quasi parton distribution function of pion
}

\author{
Nikhil Karthik ${ }^{* 1}$, Taku Izubuchi ${ }^{1,2}$, Luchang Jin ${ }^{2,3}$, Christos Kallidonis ${ }^{4}$, Swagato \\ Mukherjee $^{1}$, Peter Petreczky ${ }^{1}$, Charles Shugert ${ }^{1,4}$, Sergey Syritsyn ${ }^{2,4}$
}

${ }^{1}$ Physics Department, Brookhaven National Laboratory, Upton, NY 11973, USA

${ }^{2}$ RIKEN-BNL Research Center, Brookhaven National Lab, Upton, NY, 11973, USA

${ }^{3}$ Physics Department, University of Connecticut, Storrs, Connecticut 06269-3046, USA

${ }^{4}$ Department of Physics and Astronomy, Stony Brook University, Stony Brook, NY 11794, USA

\begin{abstract}
We present preliminary numerical results on the connected piece of the quasi-PDF of pion as determined using Wilson-Clover valence fermions on HISQ ensembles. We discuss its nonperturbative renormalization in RI/MOM scheme with and without removal of the divergent selfenergy part, and compare its running with expectation from perturbation theory. We also discuss the matching of pion QPDF to PDF, and various systematic effects associated with it.
\end{abstract}

The 36th Annual International Symposium on Lattice Field Theory - LATTICE2018

22-28 July, 2018

Michigan State University, East Lansing, Michigan, USA.

${ }^{*}$ Speaker. 


\section{Introduction}

For hard scattering processes involving hardons, such as the deep inelastic scattering of leptons on hadrons, the total cross-section becomes a convolution of the perturbatively calculable partonic cross-section and the universal, non-perturbative parton distribution function [1]. In the lightcone gauge, the parton distribution function has the familiar interpretation of the probability of finding a parton with a fraction $x$ of the energy of a fast moving hadron. Until recently, the PDFs were obtained through global fits to the experimental data $[2,3,4,5]$. Naively, one cannot use the standard lattice Monte Carlo to determine the PDFs since it involves quark and anti-quark operators separated along the light-cone, which therefore has a sign problem associated with it. Recently, the computation of PDFs using quasi-PDF, which involves equal-time Euclidean correlations of spatially separated quark-antiquark pair, of fast moving hadron and then matching to PDF using large momentum effective theory approach has been proposed to be a solution [6, 7]. It is the aim of this Lattice proceeding to present our preliminary determination of the PDF of pion using the qPDF approach as well as to discuss various systematic effects associated with this procedure. Recently, a global fit analysis of pion PDF was obtained in [8]. The reader can refer to [9] for the qPDF determination of valence PDF of pion using different lattice spacing, ensemble and matching procedure than what is used here.

Below, we outline the steps involved in the quasi-PDF approach and note the different places which can potentially lead to systematic effects that need to be controlled in the extraction of PDF:

1. First, one computes the bare quasi-PDF using the three-point to two-point function ratio given by

$$
q_{\Gamma}\left(z, P_{z} ; \Delta t\right)=\frac{\left\langle\hat{\pi}_{S}^{\dagger}\left(P_{z}, \Delta t\right) O_{\Gamma}(z) \hat{\pi}_{S}\left(P_{z}, 0\right)\right\rangle}{\left\langle\hat{\pi}_{S}^{\dagger}\left(P_{z}, \Delta t\right) \hat{\pi}_{S}\left(P_{z}, 0\right)\right\rangle},
$$

where $\hat{\pi}_{S}\left(P_{z}, t\right)$ is the pion operator inserted at time-slice $t$ with the spatial momentum $\left(0,0, P_{z}\right)$ and smeared using a method $S, \Delta t$ is the source-sink separation and the bilocal qPDF operator with Dirac structure $\Gamma$ is given by

$$
O_{\Gamma}(z ; \tau)=\bar{u}(x) \Gamma W_{x, x+z z} u(x+z \hat{z}),
$$

where $W$ is the Wilson from $x$ to $x+z \hat{z}$. There is both a connected and a disconnected piece to the above matrix element. Here, we only focus on the connected part of this. The presence of finite $\Delta t$ is one systematic effect in the calculation. This is discussed in more detail in the accompanying Lattice proceeding.

2. Then, one renormalizes the bare quasi-PDF using nonperturbative renormalization (NPR) procedure. Here, we use the RI-MOM renormalization condition with $\not p$ projection [10]

$$
q_{\gamma_{t}}^{R}\left(z, P_{z} ; p^{R}, \Delta t\right)=Z_{\gamma_{t} \gamma_{t}}\left(z, p^{R}\right) q_{\gamma_{t}}\left(z, P_{z} ; \Delta t\right)
$$

with the renormalization constant $Z\left(z, p^{R}\right)$ determined using the following condition imposed on the amputated quark qPDF, $\Lambda(z, p)$ using external quark states at momentum $p=p^{R}$ :

$$
\operatorname{Tr}\left(\not p_{R} \Lambda\left(z, p_{R}\right)\right)=12 p_{t}^{R} e^{-i p_{z}^{R} z} .
$$


In the case of $\Gamma=\gamma_{z}$, one should also take care of mixing between the bare $\Gamma=\gamma_{z}$ and $\Gamma=1 \mathrm{qPDFs}$ [11]. The running of the renormalized quark qPDF is simply the dependence of the renormalized amputated quark qPDF $\Lambda\left(z, p ; p^{R}\right)$ on $p$, with $p$ slightly away from $p^{R}$. Any mismatch between the nonperturbative and 1-loop perturbative running could be another source of systematic error.

3. The renormalized real space qPDF $q_{\gamma_{t}}^{R}\left(z, P_{z} ; p^{R}, \Delta t\right)$ is Fourier transformed to $\tilde{q}_{\gamma_{t}}\left(x, P_{z} ; p^{R}, \Delta t\right)$, where $x$ is the momentum fraction of $P_{z}$. That is

$$
\tilde{q}_{\gamma_{t}}\left(x, P_{z} ; p^{R}, \Delta t\right)=2 E\left(P_{z}\right) \int_{-\infty}^{\infty} \frac{d z}{4 \pi} q_{\gamma_{t}}^{R}\left(z, P_{z} ; p^{R}, \Delta t\right) e^{-i x P_{z} z},
$$

where $E\left(P_{z}\right)$ is the energy. In order to take the Fourier transform, the data has to be interpolated by a continuous curve. More importantly, one has actual data for the real space qPDF only over a certain finite range of $z$. In this case, the absence of data for larger $z$ and what one does to take care of this could lead to further systematic uncertainties.

4. The final step is the matching of the Fourier transform of the renormalized qPDF to PDF at a factorization scale $\mu[6,12]$. For this, we convolute $\tilde{q}(x)$ with the matching coefficient. In principle, the effect of this procedure should completely remove any dependence on $p^{R}$ and transmute it to a dependence on $\mu$.

We present results using the a $=0.06 \mathrm{fm}$, HISQ sea quark ensemble generated by the HotQCD collaboration. On this, we use 1-HYP smeared Wilson-clover valence quarks to determine the qPDF. More details on the measurement techniques is given in another proceeding accompanying this one.

\section{Effectiveness of perturbation theory to describe NPR}
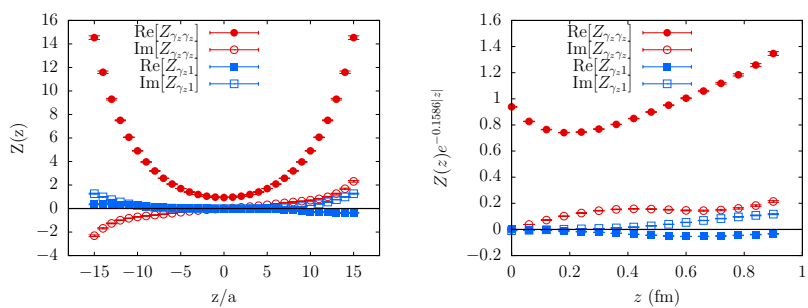

Figure 1: Renormalization constants $Z_{\Gamma \Gamma^{\prime}}$ including (left) and without including (right) the linearly divergent self-energy contribution from the Wilson line connecting the quark and anti-quark. In the two panels, the data for the diagonal $Z_{\gamma_{2}, \gamma_{z}}$ as well as the mixing with the scalar $Z_{\gamma_{2}, 1}$ are shown. The solid symbols are for their real parts while the open ones for their imaginary parts.

The only non-perturbative ingredient that is supposed to go into the PDF computation is the projection to pion state itself, which is taken care of by using the pion source and sink at large separations. The other computations, including the renormalization of the real space qPDF in the presumably relevant range of $|z|<1 \mathrm{fm}$ and the subsequent matching should be perturbative. 
The subtlety with regard to the renormalization constant is the presence of divergent self-energy contribution, $e^{c|z|}$, in the bare Wilson line $W_{x, x+z \hat{z}}$ connecting the quark and anti-quark. This should not be problem for perturbative calculation since this multiplicative factor should cancel between the NPR Z-factor and the bare qPDF. However, we check that what remains after the subtraction of the self-divergent piece is $O(1)$ as one would expect from perturbative calculation. For the one-level HYP smeared link, we use the nonperturbatively determined value $c=0.1586$ [13]. In Figure 1, we show the the diagonal renormalization factor $Z_{\gamma_{z}, \gamma_{z}}$ as well as the offdiagonal $Z_{\gamma_{t}, \gamma_{t}}$ as a function of $z$. The red points are the real parts while the blue ones are the imaginary parts. In the left panel, we show the entire renormalization factor. In the right panel, we show the renormalization factors after multiplying by $e^{-c|z|}$. As one can see, the renormalization factor after the subtraction of the divergent piece, $Z_{\gamma_{z} \gamma_{z}} e^{-c|z|}$, is surprisingly close to one even for distances upto $1 \mathrm{fm}$. We see a similar behavior for $Z_{\gamma_{t} \gamma_{t}}$ as well.

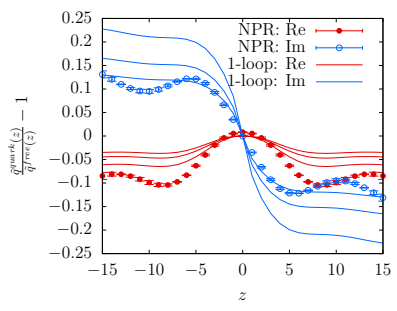

Figure 2: Comparison of quark qPDF to perturbation theory. Refer text below for explanation.

In order to see if we see a quantitative agreement of the $Z$ factors with perturbative calculation, we look for the running of

$$
\zeta(z ; p)=\frac{\operatorname{Tr}\left(\not p \Lambda^{R}\left(p ; p_{R}\right)\right)}{\operatorname{Tr}\left(\not p \Lambda^{R}\left(p_{R} ; p_{R}\right)\right)}-1,
$$

where $\Lambda^{R}\left(p ; p_{R}\right)$ is the amputated qPDF with momentum $p$ and renormalized at momentum $p^{R}$. The term $\operatorname{Tr}\left(\not p \Lambda^{R}\left(p_{R} ; p_{R}\right)\right)$ is the free field value by the renormalization condition. Since the corresponding expression for $\zeta$ from perturbation theory is simpler when $p_{z}^{R}=p_{z}$, we study this case here. In Figure 2, we show the real and imaginary parts of $\zeta(z ; p)$ as a function of $z$ for $p=$ $(0.86,0.86,1.61,0.86) \mathrm{GeV}$ with the renormalization condition set at $p_{R}=(1.28,1.28,1.61,1.28)$ $\mathrm{GeV}$. The three curves are the corresponding 1-loop perturbative results with the strong coupling $\alpha_{S}$ determined at $2 p^{R}, p^{R}$ and $p^{R} / 2$ respectively in order to quantify the uncertaintly in the scale to be used in the 1-loop calculation. While the agreement between the data and the curves is good for the imaginary part for $|z|<0.5$, only a qualitative agreement is seen in the case of the real part. However, this $p$ dependence is only sub-leading in the case of the real part, and hence this disagreement might not be an issue given other uncertainties.

\section{3. qPDF and PDF of pion}

In Figure 3, we show the bare qPDF (open symbols) and the corresponding renormalized qPDF (filled symbols) as a function of $z$. The red points are the real parts and the blue ones are the imaginary parts. The effect of renormalization is to lift the exponentially suppressed bare qPDF at 


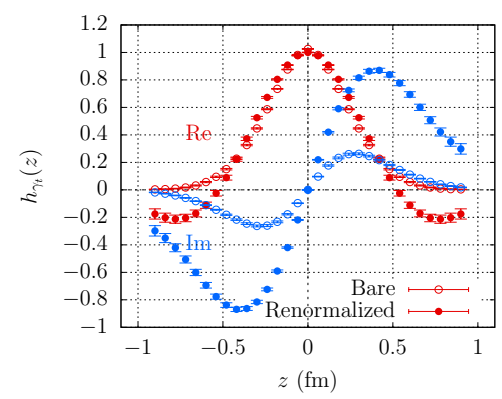

Figure 3: The real space qPDF is shown as a function of $z$. The real and imaginary parts are shown using red and blue points respectively. The open symbols are for the bare qPDF and the solid ones are for the renormalized qPDF.

larger $z$. This effect is more significant in the imaginary part than in the real part of qPDF. We have data for qPDF from $z / a=-15$ to 15 in the above plot which ranges from $-1 \mathrm{fm}$ to $1 \mathrm{fm}$ in physical units.
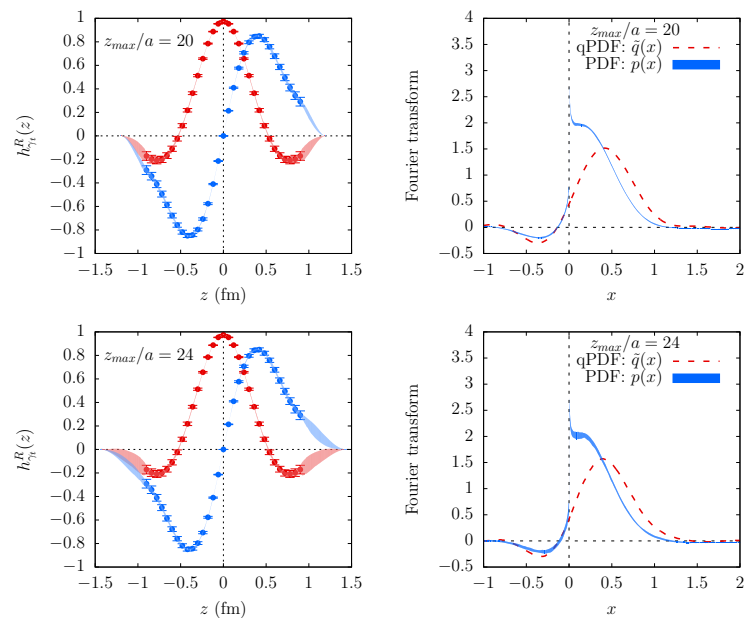

Figure 4: Cubic interpolation and extrapolation to $z_{\max } / a=20$ (top-left) and $z_{\max } / a=24$ (bottom-left) are shown. The corresponding Fourier transform of qPDF and the matched PDF at $\mu=3.2 \mathrm{GeV}$ are shown in the right panels. The description of the plots is in the text below.

In order to take the Fourier transform, we interpolate the real and imaginary parts of $q^{R}(z)$ using cubic spline. To quantify the effect of $q^{R}(z)$ at larger $z$ where we do not have data currently, we extend the spline to an arbitrary value of $|z|=z_{\max }$, at which point we set the spline and its derivative to 0 . By varying the value of $z_{\max }$, we quantify the effect of large distance part of qPDF on the extracted PDF. It is the expectation that the large distance behavior of qPDF should not affect the short distance physics of PDF. The top-left panel of Figure 4 shows the real space real and imaginary parts of qPDF, and the cubic interpolation (bands) which is extended upto $z_{\max } / a=20$. The right panel shows the Fourier transform of the interpolation shown on the left, using dashed lines. The PDF obtained by applying the matching formula to the 'dashed lines' is shown using the blue $1-\sigma$ error bands. The corresponding plots for $z_{\max } / a=24$ is shown in the bottom panels. The effect of the extrapolation is mainly to increase the error in $\tilde{q}(x)$ and matched PDF $p(x)$ without 
affecting the central values. This is reassuring that the unknown long distance contribution of qPDF does not play a significant role. However, this could still be specific to the way we are taking care of the long distance behaviour of $q(z)$. It needs to be seen if this independence of long distance physics is seen with other models of extrapolation to larger $|z|$.
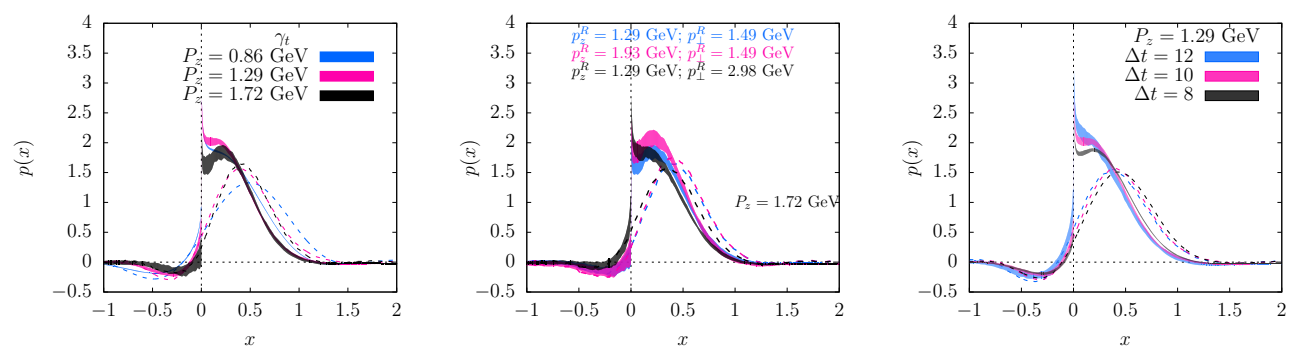

Figure 5: Matched valence PDF of pion. In all the panels, the bands are the $1-\sigma$ error bands for the PDF while the dashed curves of the same color are the corresponding $\tilde{q}_{\gamma_{t}}(x)$ used to obtain the PDF. (Left) The $P_{z}$ dependence of PDF is shown. (Center) The dependence of PDF on the renormalization scale for the qPDF is shown. (Right) The dependence on source-sink separation $\Delta t$ is shown.

Now we focus on the matched PDF that we obtain at a scale $\mu=3.2 \mathrm{GeV}$ using perturbative matching with the strong coupling $\alpha_{S}(\mu)=0.234$. The first systematic is the approach to $P_{z} \rightarrow \infty$ limit. We use $P_{z}=0.86 \mathrm{GeV}, 1.29 \mathrm{GeV}$ and $1.72 \mathrm{GeV}$, which are smaller compared to the lattice spacing of $3.28 \mathrm{GeV}$ and much larger compared to the pion mass of $0.3 \mathrm{GeV}$. In the leftmost panel of Figure 5, we show $\tilde{q}\left(x, P_{z} ; p_{z}^{R}=1.28 \mathrm{GeV}, p_{\perp}^{R}=2.21 \mathrm{GeV}\right)$ and the matched PDF $p(x, \mu=3.2 \mathrm{GeV})$, at different $P_{z}$ with every other parameter held fixed. There seems to be convergence by looking at the consistency between the $P_{z}=1.29$ and $1.72 \mathrm{GeV}$ results for the PDF. It is also reassuring that the matched PDF vanishes for $x>1$. For $|x|<\Lambda_{\mathrm{QCD}} / P_{z}$, the long distance non-perturbative effects would become important. For the largest momentum $\Lambda_{\mathrm{QCD}} / P_{z} \approx 0.1$. In the middle panel of Figure 5, we show the dependence of the matched PDF on the renormalization scale of the qPDF. The different bands correspond to different renormalization points $\left(p_{z}^{R}, p_{\perp}^{R}\right)$ as specified. The matching procedure should remove any $p^{R}$ dependence present in $\tilde{q}(x)$. A mild $p^{R}$ dependence is seen in the matched PDF data at the largest momentum consistent with the expectation. In the rightmost panel of Figure 5, we show the effect of source-sink separation $\Delta t$ on the matched PDF at fixed values of $P_{z} \mathrm{GeV}, p^{R}$ and $z_{\max }$. There is a tendency for the data in the range $x>1$ to move closer to zero while the data in the range $0<x<0.5$ to increase as the source-sink separation is increased from 8 lattice units to 12 , thereby moving towards the phenomenological expectation.

\section{Conclusion}

We presented results on the non-perturbative renormalization constants and showed that they are of order 1 after the removal of the self energy divergence of the Wilson line even upto quark antiquark separations of $1 \mathrm{fm}$. But, on a quantitative level, a slight disagreement with the perturbative prediction of the running of the quark qPDF was seen. Perhaps, this slight disagreement is not of consequence given other sources of uncertainties. Then, we presented the fourier transform of the 
connect part of the pion quasi PDF and its mild dependence on the behaviour of qPDF for distances greater than $1 \mathrm{fm}$. Finally, we presented the matched PDF of pion and discussed its dependence on the pion momentum, the renormalization scale of the qPDF and the source-sink separation.

The work was supported by the U.S. Department of Energy, Office of Nuclear Physics through the Contract No. DE-SC001270, BNL LDRD project No. 16-37, and Scientific Discovery through Advance Computing (ScIDAC) award "Computing the Properties of Matter with Leadership Computing Resources". SS also acknowledges support by the RHIC Physics Fellow Program of the RIKEN BNL Research Center. Computations were carried out using USQCD resources at JLab and BNL. This research also used an award of computer time provided by the INCITE program at the Oak Ridge Leadership Computing Facility, which is a DOE Office of Science User Facility supported under Contract DE-AC05-00OR22725.

\section{References}

[1] R. P. Feynman, Very high-energy collisions of hadrons, Phys. Rev. Lett. 23 (1969) 1415-1417.

[2] A. D. Martin, W. J. Stirling, R. S. Thorne and G. Watt, Parton distributions for the LHC, Eur. Phys. J. C63 (2009) 189-285, [0 901.0002 ].

[3] P. M. Nadolsky, H.-L. Lai, Q.-H. Cao, J. Huston, J. Pumplin, D. Stump et al., Implications of CTEQ global analysis for collider observables, Phys. Rev. D78 (2008) 013004, [0 802.0007 ].

[4] NNPDF collaboration, R. D. Ball, L. Del Debbio, S. Forte, A. Guffanti, J. I. Latorre, A. Piccione et al., A Determination of parton distributions with faithful uncertainty estimation, Nucl. Phys. B809 (2009) 1-63, [0808.1231].

[5] ZEUS, H1 collaboration, F. D. Aaron et al., Combined Measurement and QCD Analysis of the Inclusive e+- p Scattering Cross Sections at HERA, JHEP 01 (2010) 109, [0 911.088 4].

[6] X. Ji, Parton Physics on a Euclidean Lattice, Phys. Rev. Lett. 110 (2013) 262002, [1305 . 1539].

[7] X. Ji, Parton Physics from Large-Momentum Effective Field Theory, Sci. China Phys. Mech. Astron. 57 (2014) 1407-1412, [1404.6680].

[8] P. C. Barry, N. Sato, W. Melnitchouk and C.-R. Ji, First Monte Carlo Global QCD Analysis of Pion Parton Distributions, Phys. Rev. Lett. 121 (2018) 152001, [1804.01965].

[9] J.-W. Chen, L. Jin, H.-W. Lin, Y.-S. Liu, A. Schäfer, Y.-B. Yang et al., First direct lattice-QCD calculation of the $x$-dependence of the pion parton distribution function, 1804.01483.

[10] J.-W. Chen, L. Jin, H.-W. Lin, Y.-S. Liu, Y.-B. Yang, J.-H. Zhang et al., Lattice Calculation of Parton Distribution Function from LaMET at Physical Pion Mass with Large Nucleon Momentum, 1803.04393.

[11] M. Constantinou and H. Panagopoulos, Perturbative renormalization of quasi-parton distribution functions, Phys. Rev. D96 (2017) 054506, [1705.11193].

[12] I. W. Stewart and Y. Zhao, Matching the quasiparton distribution in a momentum subtraction scheme, Phys. Rev. D97 (2018) 054512, [1709.04933].

[13] TUMQCD collaboration, A. Bazavov, N. Brambilla, P. Petreczky, A. Vairo and J. H. Weber, Color screening in (2+1)-flavor QCD, Phys. Rev. D98 (2018) 054511, [1804.10600]. 